\title{
Deterioro ambiental y micromamíferos durante el Holoceno en el nordeste de la estepa patagónica (Argentina)
}

\author{
Environmental deterioration and micromammals during the Holocene in northeastern \\ Patagonian steppe (Argentina)
}

ULYSES F. J. PARDIÑAS ${ }^{1}$, GERMAN J. MOREIRA ${ }^{2.3}$, CESAR M. GARCIA-ESPONDA ${ }^{3}$ \& LUCIANO J. M. DE SANTIS ${ }^{3}$

\begin{abstract}
'Departamento Científico Paleontología Vertebrados, Museo de La Plata, Paseo del Bosque s/n, 1900 La Plata, Argentina (CONICET), email: ulyses@museo.fcnym.unlp.edu.ar

${ }^{2}$ Comisión de Investigaciones Científicas de la provincia de Buenos Aires (CIC), ${ }^{3}$ Cátedra de Anatomía Comparada, Facultad de Ciencias Naturales y Museo, Paseo del Bosque s/n, 1900 La Plata, Argentina
\end{abstract}

\section{RESUMEN}

Se estudió la composición taxonómica cualitativa y cuantitativa de una muestra de micromamíferos proveniente del paraje Lle cul $\left(43^{\circ} 20^{\prime} \mathrm{S}, 65^{\circ} 35^{\prime} \mathrm{W}\right)$, en el valle del río Chubut (Argentina), con una antiguiedad de $1830 \pm 70$ años radiocarbónicos AP. La asociación está integrada por roedores sigmodontinos (6 especies), caviomorfos (dos especies), quirópteros (dos especies) y marsupiales marmosinos (dos especies), destacándose por su abundancia Calomys cf. $C$. laucha-C. musculinus $(35,2 \%)$ y Eligmodontia sp. $(33,4 \%)$. El estudio de la frecuencia esqueletaria y marcas de corrosión sobre los restos óseos indican que el agregado ha sido generado por una estrigiforme poco destructiva, quizás Tyto alba, y depositado probablemente en época invernal. La comparación con los micromamíferos de una muestra actual de egagrópilas de $T$. alba de la misma localidad (correspondiente a un año), revela una disminución importante en la diversidad específica, con una dominancia cercana al $95 \%$ para Calomys cf. C. laucha-C. musculinus. La discusión efectuada a base de muestras actuales representativas de diferentes distritos fitogeográficos patagónicos y evidencias paleoambientales para el Holoceno superior, indican como causas más probables de este empobrecimiento al impacto antrópico durante los últimos 100 años. Actividades como la expansión de agroecosistemas habrían favorecido la dispersión y abundancia de Calomys en detrimento de otros micromamíferos.

Palabras clave: roedores, Patagonia, Holoceno, diversidad, impacto antrópico.

\section{ABSTRACT}

The qualitative and quantitative taxonomic composition of a micromammal sample from Lle cul $\left(43^{\circ} 20^{\prime} \mathrm{S}, 65^{\circ} 35^{\prime} \mathrm{W}\right)$, in the lower valley of the Chubut River (Argentina), was analyzed. The age of the sample was $1,830 \pm 70 \mathrm{yr} B P$. The association includes 6 species of sigmodontine rodents, two species of caviomorphs, two species of chiropterans, and two species of marmosine marsupials, including Calomys cf. C. laucha-C. musculinus $(35.2 \%)$ and Eligmodontia sp. $(33.4 \%)$ as the most abundant taxa. The analysis of frequency and corrosive marks on the skeletal remains indicates that the material was probably deposited during winter time by a strigiform bird, possibly Tyto alba, which explains the low level of bone corrosion. Comparison with a recent sample of pellets from Tyto alba from the same locality, in which Calomys cf. C. laucha-C. musculinus accounted for $95 \%$ of the total micromammals, shows a marked loss in today's species diversity. Comparison with modern samples of owl pellets from different Patagonian phytogeographic districts, together with paleoenvironmental evidence from the Late Holocene, indicate that human activities are probably the most important agent of the modification of the environment. Activities like overgrazing by sheep and expansion of agriculture may have aided both the dispersal as well as the increased present-day abundance of Calomys on account of another micromammals.

Key words: rodents, Patagonia, Holocene, diversity, anthropic impact.

\section{INTRODUCCION}

La Patagonia es uno de los pocos territorios continentales extra-antárticos extendido más allá de los $40^{\circ}$ Sur que sostiene comunidades bióticas complejas. Sus particulares características fisiográficas, climáticas y biogeográficas han atraído la atención de numerosos naturalistas desde comienzos del siglo pasado. En los últimos años se ha incrementado nuestro conocimiento de 
los mamíferos patagónicos actuales (véase Monjeau et al. 1994, Pearson 1995) y de las condiciones paleoambientales inferidas para el Pleistoceno más tardío y Holoceno. Sin embargo, gran parte de la información obtenida sobre este último aspecto proviene de análisis paleopalinológicos y de evidencias estratigráficas del sector andino, tanto en la región norpatagónica como en el extremo sur (véase Clapperton 1993 y las referencias allí citadas).

En gran medida, la denominada Patagonia extra-andina o "estepa patagónica" en sentido amplio, es prácticamente desconocida desde un punto de vista paleoambiental (véase Iriondo 1995). Esto puede atribuirse, en parte, a lo extenso de los territorios involucrados -principalmente mesetas y escudos basálticos y planicies de rodados- pero más seguramente a la falta o dificultad en encontrar archivos paleoclimáticos naturales (i.e., polen, insectos, turba, egagrópilas, etc.). Unos pocos trabajos han tratado de esclarecer las variaciones climático-ambientales para los últimos 10.000 años, en buena medida utilizando indicadores polínicos y macrobotánicos (Prieto \& Stutz 1996, Markgraf 1993, Markgraf et al. 1995, Heusser et al. 1988, Villalba 1995), faunísticos (Miotti 1993, Pardiñas 1998, Pearson \& Pearson 1993, Simonetti \& Rau 1989) o geomorfológicosestratigráficos (Stine \& Stine 1990, Galloway et al. 1988). En cuanto a la estepa patagónica nordeste y central, la situación es aún más crítica, existiendo sólo una pocas contribuciones (Schäbitz 1991, 1994, Garleff et al. 1994).

La comparación entre muestras actuales y fósiles con el objetivo de estimar la signifación paleoambiental de estas últimas, requiere de un análisis tafonómico que permita inferir los factores causales de la acumulación de los restos. Esto permite conocer con mejor detalle los sesgos que están afectando la estructura taxonómica cuantitativa y cualitativa de la muestra en estudio y no otorgar valor paleoecológico a características emergentes de procesos bioestratinómicos y/o diagenéticos (véase Andrews 1990, Stahl 1996).

Los objetivos de este trabajo son: a) la caracterización taxonómica cualitativa y cuantitativa de una muestra de micromamíferos fósiles, principalmente roedores sigmodontinos, proveniente de sedimentos holocénicos del nordeste patagónico; b) el análisis de los atributos tafonómicos de la misma a fin de inferir el agente responsable de la acumulación de los restos óseos y c) a través de la comparación con muestras de roedores actuales, una interpretación de las modificaciones globales del paisaje ocurridas en los últimos dos mil años -principalmente a nivel de comunidades florísticas- que configuraron la actual comunidad de micromamíferos.

La hipótesis de trabajo empleada supone que uno de los efectos del deterioro ambiental por causas antrópicas en un área dada es la pérdida de la diversidad específica. En este sentido se observarán variaciones en la diversidad de micromamíferos entre muestras fósiles y actuales generadas por aves rapaces, reflejadas -en estas últimastanto en un empobrecimiento de la riqueza de presas consumidas como en un aumento de la dominancia de ciertos taxones oportunistas.

\section{MATERIALES Y METODOS}

La muestra de micromamíferos fósiles estudiada fue coleccionada en Lle cul $\left(43^{\circ} 20^{\prime} \mathrm{S}, 65^{\circ} 35^{\prime} \mathrm{O}\right)$, paraje situado $10 \mathrm{~km}$ al sudoeste de la localidad de Gaiman, en el valle del río Chubut (Fig. 1). Los restos óseos rellenaban una grieta, sellada cuspidalmente por yeso. Una datación radiocarbónica sobre la fracción de colágeno contenida en el material postcraneano brindó $1.830 \pm 70$ años AP (LP-578), lo que permite referir la muestra al Holoceno superior. Las determinaciones taxonómicas se efectuaron sobre restos craneanos y mandibulares; el criterio sistemático expresado sigue aquel de Galliari et al. (1996). La frecuencia específica se estimó mediante el cálculo del número de especímenes (NISP) y del número mínimo de individuos (MNI) (Grayson 1973). El material estudiado está depositado en la Cátedra de Anatomía Comparada (Facultad de Ciencias Naturales y Museo, Universidad Nacional La Plata).

El agregado fósil de Lle cul presentaba un aspecto que a priori hacía suponer su origen en egagrópilas de aves rapaces: una acumulación densa de restos óseos craneanos y postcraneanos en buen estado de conservación. La contrastación de dicha hipótesis proviene de dos fuentes de evidencias tafonómicas: análisis de frecuencias esqueletarias y relevamientos de marcas de corrosión (principalmente debidas al ataque de los ácidos digestivos) sobre los restos óseos (Andrews 1990, Hoffman 1988, Stahl 1996). Se trabajó con una muestra conformada por un NISP de 3110 y un MNI de 339, a la que se le calcularon las frecuencias esqueletarias absolutas y la abundancia relativa sobre la base del NISP (Andrews, 1990: 45). Debido al bajo porcentaje de fracturas no se realizaron las correcciones propuestas por Lyman (1994) entre el NISP y número mínimo de elementos (MNE). La muestra fósil fue comparada -a los fines de establecer el proceso de acumu- 
lación de los restos-con agregados actuales generados por Tyto alba, Asio flammeus y Bubo bubo (sobre datos de Andrews, 1990: tabla 12) y con una muestra de egagrópilas actuales disgregadas de $T$. alba de Patagonia.

Para inferir el período del año en el cual se depositó la muestra fósil se construyeron pirámides etarias de Calomys, agrupando los individuos en tres clases de edad relativa (juveniles, adultos y seniles) sobre la base del desgaste de los molares (véase Bellocq \& Kravetz 1983). Esta metodología presenta una serie de problemas cuando se compara con la estructura de edades obtenida mediante datos de trampeo (véase Pardiñas 1998), debido a que implica asumir que las aves rapaces depredan en forma directamente proporcional a la abundancia de cada clase de edad por estación. Sin embargo, algunas evidencias indican que las estrigiformes realizan una depredación selectiva por tamaño y, por ende, por edad (véase Colvin \& McLean 1986, Jaksic \& Yáñez 1979, Faverín 1987, Zamorano et al. 1986, contra Saint Girons 1973). Este hecho trae aparejado una distorsión en las pirámides etarias resultantes del análisis de egagrópilas planteando una nota de incertidumbre en cuanto a su real valor para estimar estacionalidad. Un segundo aspecto, aunque ya inherente a las características del registro fósil,

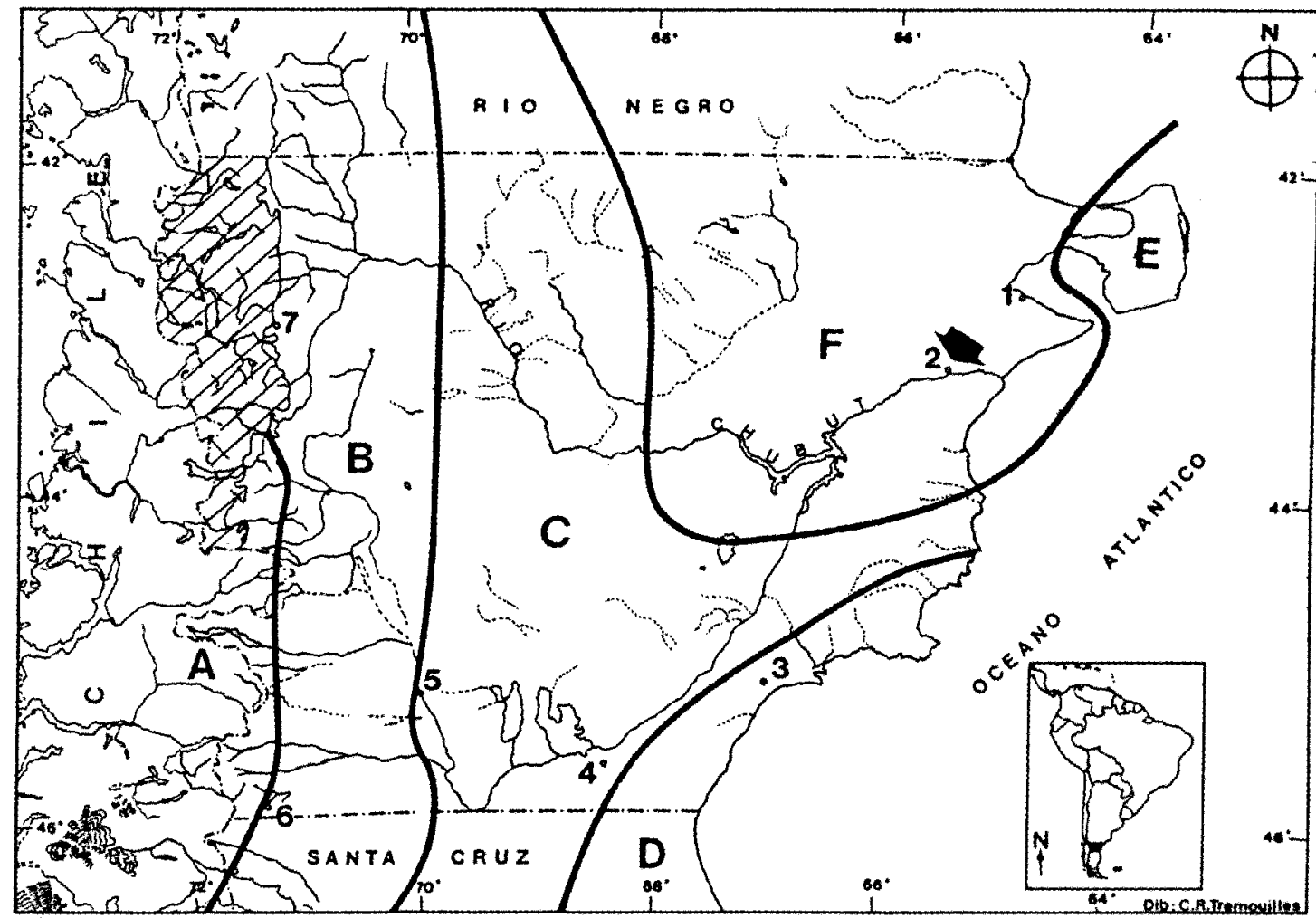

Fig. I. Mapa de la provincia de Chubut con indicación de la localidad de procedencia de la muestra fósil estudiada (flecha) y de las muestras actuales utilizadas en el análisis comparativo. Se indican, asimismo, las divisiones fitogeográficas de la estepa patagónica (en rayado el área ocupada por los bosques andinos) sobre la base del esquema de Soriano (1956a). Referencias: Provincia Patagónica, A. Distrito subandino, B. Distrito occidental, C. Distrito central y D. Distrito del Golfo San Jorge; F. Provincia del Monte; E. Región transicional con el Monte; Localidades: 1. Laguna Blanca y Punta Este (De Santis \& Pagnoni 1989), 2. Lle cul (este trabajo y García Esponda et al. 1998), 3. Estancia El Gauchito (Pardiñas datos inéditos), 4. Valle Hermoso (Massoia \& Pardiñas 1988), 5. Meseta Lehman (De Santis et al. 1996), 6. Río Corintos (De Santis et al. 1993) y 7. Trevelin (De Santis et al. 1991, 1994).

Map of Chubut Province showing the localities of fossil sample (arrow) and recent samples discussed in this paper. Major vegetation units of Patagonian steppe (shaded area: Andean forests) following Soriano (1956a). Patagonian Province: A. Subandean district, B. Occidental district, C. Central district, and D. Golfo San Jorge district; F. Monte Province; E. Transitional region with Monte Province; Localities: 1. Laguna Blanca y Punta Este (De Santis \& Pagnoni 1989), 2. Lle cul (this paper and García Esponda et al. 1998), 3. Estancia El Gauchito (Pardiñas unplublished data), 4. Valle Hermoso (Massoia \& Pardiñas 1988), 5. Meseta Lehman (De Santis et al. 1996), 6. Río Corintos (De Santis et al. 1993), and 7. Trevelin (De Santis et al. 1991, 1994). 
es la posibilidad de mezcla de agregados producidos en diferentes estaciones. Una manera de superar el primer factor es comparar, al igual que en la reconstrucción paleoambiental, pirámides etarias entre muestras fósiles y egagrópilas actuales (toda vez que se demuestre que el agregado fósil tiene un origen en la actividad depredadora de aves rapaces). Esta metodología precisa conocer la estación en la que fueron expelidos los regurgitados actuales. Asimismo, pirámides etarias coincidentes en más de un taxón, brindan mayor confiabilidad a la estimación.

La riqueza y diversidad específicas de la muestra fósil fueron comparadas con las calculadas en una muestra actual de egagrópilas de $T$. alba (representativa de un año) para la misma localidad, según datos de García-Esponda et al. (1998). Esta misma muestra se utilizó para estimar la estacionalidad. Una comparación regional fue efectuada con los micromamíferos de la totalidad de los análisis de egagrópilas de $T$. alba conocidos para la provincia de Chubut (Fig. 1 y Tabla 1), correspondientes a los diferentes distritos fitogeográficos en que se divide la Patagonia (véase Soriano 1956a, Soriano et al. 1983, Beeskow et al. 1987). Se confeccionaron matrices de presencia-ausencia y frecuencias específicas absolutas (esta última transformada por el método "octavas", véase Gauch 1982) por cada muestra y se compararon mediante el coeficiente "Jaccard" y "distancia euclideana", respectivamente. Las relaciones se expresaron gráficamente mediante fenogramas construidos por la técnica UPGMA (véase Crisci \& López Armengol 1983), calculándose el coeficiente de correlación cofenética (CCC) como una medida de la distorsión de los datos al ser forzados en agrupamientos. Estas operaciones se efectuaron con el software de análisis multivariado NTSYS-pc (Rolfh 1993). Los fenogramas fueron obtenidos mediante 999 iteraciones. No se aplicaron pruebas de significación específicas para cada agrupamiento (véase Strauss 1982); los elevados valores de CCC implican una baja distorsión de la matriz original,

TABLA 1

Frecuencia de micromamíferos (expresada en MNI) en egagrópilas de Tyto alba de diferentes localidades de la provincia de Chubut, Argentina, utilizadas en la comparación con la muestra fósil de Lle cul (referencias en Fig. 1)

Data matrix (micromammals frequency as MNI) for comparison between the fossil sample from Lle cul (Chubut, Argentina) and the analysis of recent owl pellets of Tyto alba from Chubut Province (references in Fig. 1)

\begin{tabular}{|c|c|c|c|c|c|c|}
\hline Taxón & $\begin{array}{l}\text { Laguna Blanca } \\
\text {-Punta Este }\end{array}$ & Trévelin & Río Corintos & $\begin{array}{l}\text { Meseta } \\
\text { Lehman }\end{array}$ & $\begin{array}{c}\text { Valle } \\
\text { Hermoso }\end{array}$ & $\begin{array}{c}\text { Estancia } \\
\text { El Gauchito }\end{array}$ \\
\hline
\end{tabular}

\begin{tabular}{|c|c|c|c|c|c|c|}
\hline Abrothrix longipilis & & 24 & & & & \\
\hline Abrothrix olivaceus & & 119 & 19 & & & \\
\hline Abrothrix sp. & & & & & & 86 \\
\hline Akodon molinae & 8 & & & & & \\
\hline Akodon nucus & 11 & & & & & \\
\hline Akodon sp. & & & & 1 & & \\
\hline Calomys sp. & & & & 77 & 1 & \\
\hline Chelemys macronyx & & 1 & 1 & & & \\
\hline Ctenomys sp. & 4 & & & 1 & 1 & \\
\hline Eligmodontia sp. & 187 & 142 & 45 & & 1 & . 12 \\
\hline Euneomys chinchilloides & & & & & & 21 \\
\hline Geoxus valdivianus & & 1 & 2 & & & \\
\hline Graomys griseoflavus & 67 & & & & 1 & 8 \\
\hline Lestodelphys halli & & & & & & 2 \\
\hline Loxodontomys micropus & & 17 & & & & \\
\hline Microcavia australis & 2 & & & & & 1 \\
\hline Oligoryzomys longicaudatus & & 132 & 4 & & & \\
\hline Phyllotis xanthopygus & & & & & 1 & 33 \\
\hline Reithrodon auritus & 10 & 108 & 2 & 1 & 1 & 42 \\
\hline Thylamys sp. & 72 & & & & & 1 \\
\hline TOTAL & 361 & 544 & 73 & 80 & 6 & 206 \\
\hline Número de especies & 8 & 8 & 6 & 4 & 6 & 9 \\
\hline
\end{tabular}


TABLA 2

Composición taxonómica cualitativa y cuantitativa para las muestras fósil y actual de Lle cul (Chubut, Argentina)

Qualitative and quantitative taxonomic composition of the fossil and recent samples from Lle cul (Chubut, Argentina)

\begin{tabular}{|c|c|c|c|c|c|c|}
\hline \multirow[b]{2}{*}{ Taxón } & \multicolumn{4}{|c|}{ Lle Cul fósil } & \multicolumn{2}{|c|}{ Le Cul actual* } \\
\hline & NISP & NISP\% & MNI & $\mathrm{MNI} \%$ & MNI & MNI\% \\
\hline Calomys cf. C.laucha-C.musculinus & 1.462 & 36,7 & 415 & 35,2 & 1.708 & 94,5 \\
\hline Eligmodontia sp. & 1.378 & 34,6 & 393 & 33,4 & 59 & 3,3 \\
\hline Oligoryzomys longicaudatus & 390 & 9,8 & 107 & 9,1 & 2 & 0,1 \\
\hline Reithrodon auritus & 297 & 7,4 & 82 & 7,0 & 4 & 0,2 \\
\hline Akodon sp. & 169 & 4,2 & 69 & 5,9 & 6 & 0,3 \\
\hline Ctenomys sp. & 161 & 4,0 & 60 & 5,1 & 8 & 0,4 \\
\hline Graomys griseoflavus & 41 & 1,0 & 14 & 1,2 & 15 & 0,8 \\
\hline Tadarida brasiliensis & 27 & 0,7 & 11 & 0,9 & - & - \\
\hline Microcavia australis & 29 & 0,7 & 10 & 0,8 & 1 & 0,1 \\
\hline Histiotus montanus & 12 & 0,3 & 6 & 0,5 & 2 & 0,1 \\
\hline Thylamys sp. & 15 & 0,4 & 5 & 0,4 & 2 & 0,1 \\
\hline Chiroptera gen. et sp. indet. & 5 & 0,1 & 5 & 0,4 & - & - \\
\hline Lestodelphys halli & 1 & 0,0 & 1 & 0,1 & - & - \\
\hline TOTAL & 3.987 & & 1.178 & & 1.807 & \\
\hline
\end{tabular}

*Datos de García-Esponda et al. (1998)

además, las iteraciones efectuadas dieron por resultado un único fenograma para cada caso estudiado. En el Apéndice se muestran las matrices de similitud y distancia.

\section{RESULTADOS}

\section{Taxonómicos}

En la Tabla 2 se presenta el NISP y MNI para cada taxón de la muestra fósil y actual de Lle cul. El listado sistemático reconocido en la muestra fósil es el siguiente:

Orden Rodentia Bowdich, 1821:

Familia Muridae Illiger, 1815:

Tribu Akodontini Vorontzov, 1959, Akodon Meyen, 1833: Akodon sp.; Tribu Oryzomyini Vorontzov, 1959, Oligoryzomys Bangs, 1900: O. longicaudatus (Benett, 1832); Tribu Phyllotini Vorontzov, 1959, Calomys Waterhouse, 1837 : Calomys cf. C. laucha-C. musculinus, Eligmodontia F. Cuvier, 1837: Eligmodontia sp., Graomys Thomas, 1916: G. griseoflavus (Waterhouse, 1837), Reithrodon Waterhouse, 1837: $R$. auritus (Fischer, 1814).

Familia Octodontidae Waterhouse, 1839:

Ctenomys Blainville, 1826: Ctenomys sp.

Familia Caviidae Waterhouse, 1839:

Microcavia $\mathrm{H}$. Gervais y Ameghino, 1880:

M. australis (I. Geoffroy y D'orbigny, 1833).
Orden Chiroptera Blumenbach, 1779 .

Familia Molossidae Gill, 1872:

Tadarida Rafinésque, 1814: T. brasiliensis (I. Geoffroy, 1824).

Familia Vespertilionidae Gray, 1821:

Histiotus Gervais, 1856: H. montanus (Philippi

y Landbeck, 1861).

Orden Didelphimorphia Illiger, 1811 :

Familia Didelphidae Gray, 1821, Lestodelphys Tate, 1934: L. halli (Thomas, 1921), Thylamys Gray, 1843: Thylamys sp.

\section{Tafonómicos}

Las frecuencias esqueletarias absolutas para cada muestra se observan en la Tabla 3. El análisis de las abundancias relativas sobre el NISP indica que las mayores similitudes se presentan entre la muestra fósil de Lle cul y la muestra de egagrópilas actuales disgregadas de $T$. alba de Patagonia $\left(\chi^{2}\right.$ $=9,74 ; \mathrm{gl}=8 ; \mathrm{P}>0,05)$. Los otros agregados muestran diferencias significativas (Lle cul fósilA. flammeus: $\chi^{2}=19,36 ; \mathrm{gl}=8 ; \mathrm{P}<0,05$; Lle cul fósil-T. alba: $\chi^{2}=23,49$; Lle cul fósil-B. $b u b o: \chi^{2}$ $=17,79)$. Los valores de abundancias relativas (Fig. 2) presentan máximos en los elementos craneanos (hemimandíbulas y hemimaxilares), húmero, fémur y, en menor medida, tibia. Estos mismos máximos se mantienen, con cierto grado de variabilidad, en los agregados actuales originados por aves estrigiformes. Es necesario tener 
en cuenta, para la evaluación de estos resultados, el impacto de los factores bioestratinómicos (exposición a la intemperie, acción de ácidos húmicos, pisoteo, destrucción por raíces, etc.) que afecta a las muestras fósiles. Además, la importante variabilidad intrínseca que han revelado los análisis de muestras actuales de $T$. alba (véase Saavedra \& Simonetti 1998).

Andrews (1990, véase también Fernández-Jalvo \& Andrews 1992) categoriza a los diferentes agentes bióticos que acumulan restos -estrigiformes, rapaces diurnas, carnívoros- según el grado de modificación de los conjuntos óseos. El relevamiento de marcas en la muestra fósil de Lle cul indica que menos del $5 \%$ del NISP total presenta corrosión por digestión, concentrada principalmente en el extremo de los incisivos (Fig. 3). Tyto alba se cuenta entre las estrigiformes que menor transformación producen sobre los huesos (Andrews 1990: 88-89).

Las evidencias analizadas sugieren que el agregado fósil de Lle cul ha sido producido por la actividad acumuladora de aves rapaces nocturnas y, muy posiblemente, por T. alba, una estrigiforme con acción destructiva moderada. Estos resultados justifican la comparación con agregados ac-

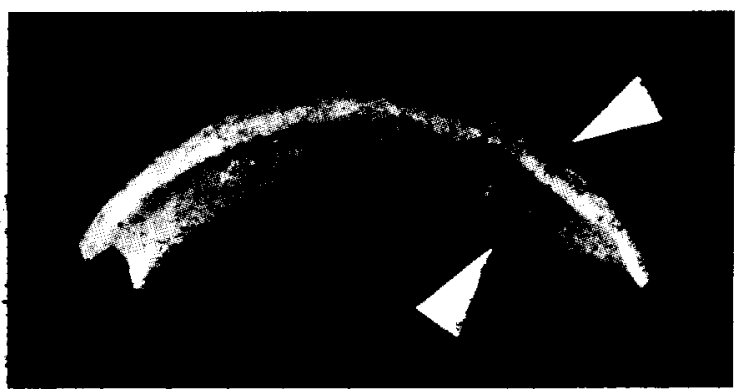

Fig. 3. Incisivo superior de Ctenomys sp. de Lle cul fósil (Chubut, Argentina) mostrando digestión moderada (sensu Fernández-Jalvo \& Andrews 1992) sobre el extremo (flechas).

Upper incisor of Ctenomys sp. from the fossil sample of Lle cul (Chubut, Argentina) showing moderate digestion (sensu Fernández-Jalvo \& Andrews 1992) on the tip (arrows).

tuales generados por lechuzas, a la hora de inferir las implicaciones ambientales de la muestra.

\section{Estacionalidad}

La estructura etaria de Calomys cf. C. laucha-C. musculinus en la muestra fósil de Lle cul (Fig. 4)

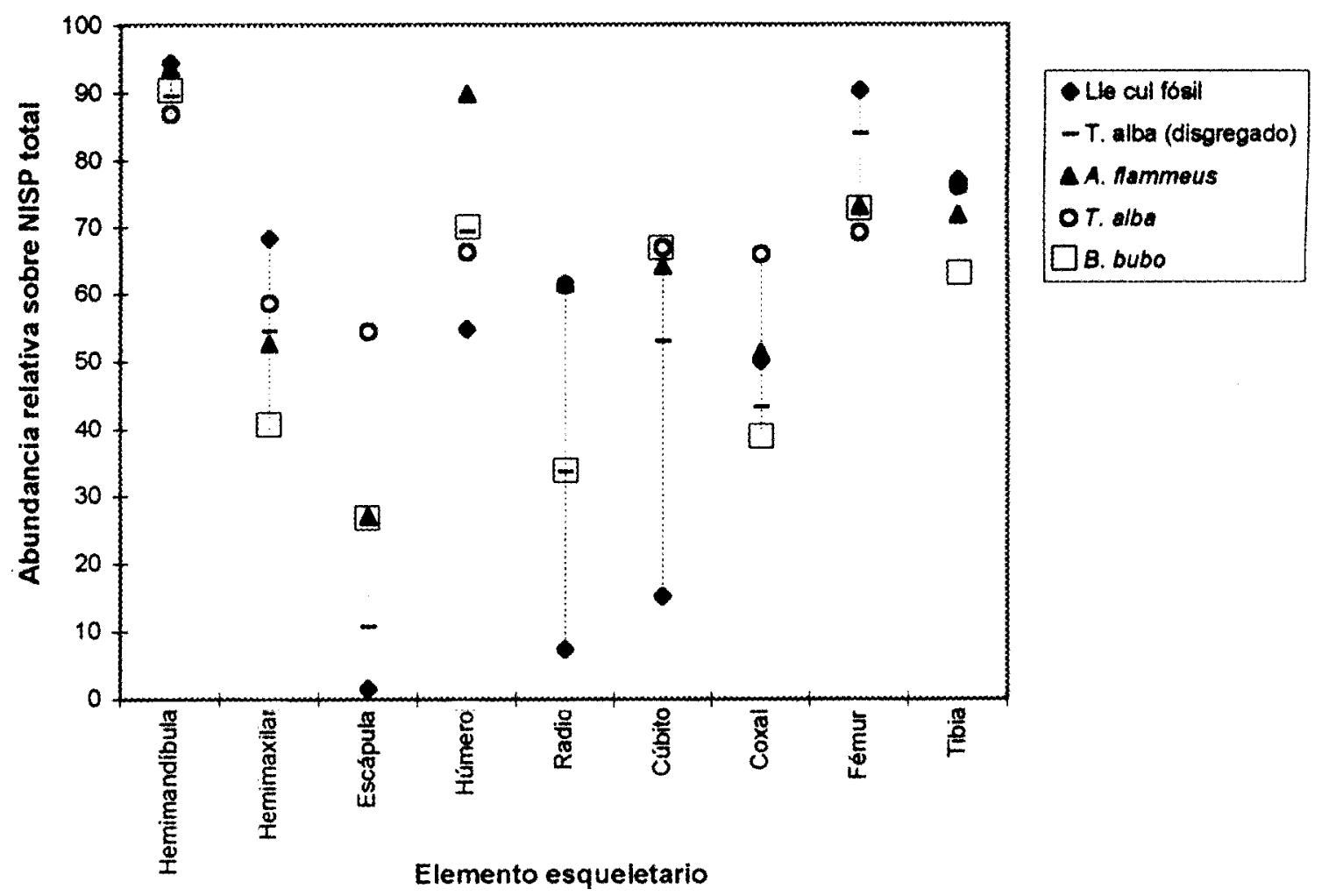

Fig. 2. Abundancias relativas de especímenes esqueletarios de la muestra fósil de Lle cul (Chubut, Argentina) y de otras muestras utilizadas en el análisis tafonómico (véase el texto).

Relative abundances of skeletal specimens comparing the fossil sample of Lle cul (Chubut, Argentina) and other samples used in the taphonomic analysis (see the text). 


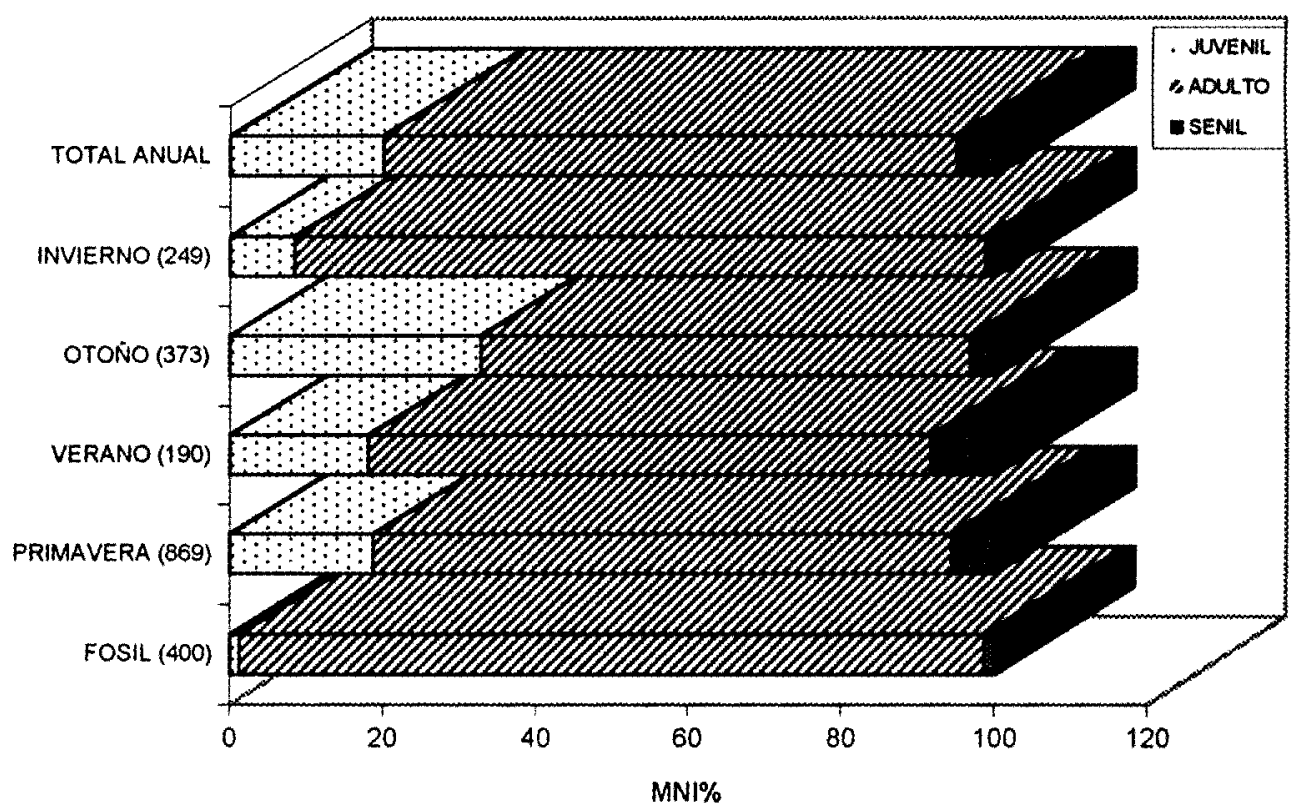

Fig. 4. Estructura etaria de Calomys cf. C. laucha-C. musculinus en la muestra fósil y en la muestra actual (por estaciones y total) de Lle cul (Chubut, Argentina). Se indican los tamaños de muestra (MNI). Age structure of Calomys cf. C. laucha-C. musculinus in the fossil and recent (by seasons and total) samples from Lle cul (Chubut, Argentina). Sample size as MNI is given.

muestra que las proporciones entre juveniles, adultos y seniles son similares a las registradas actualmente para la estación invernal en la dieta de T. alba de la misma localidad (véase GarcíaEsponda et al. 1998). Lamentablemente, la representación de Eligmodontia sp. en las muestras actuales es muy baja (Tabla 2) e impide contrastar la pirámide etaria de este roedor con la del conjunto fósil.

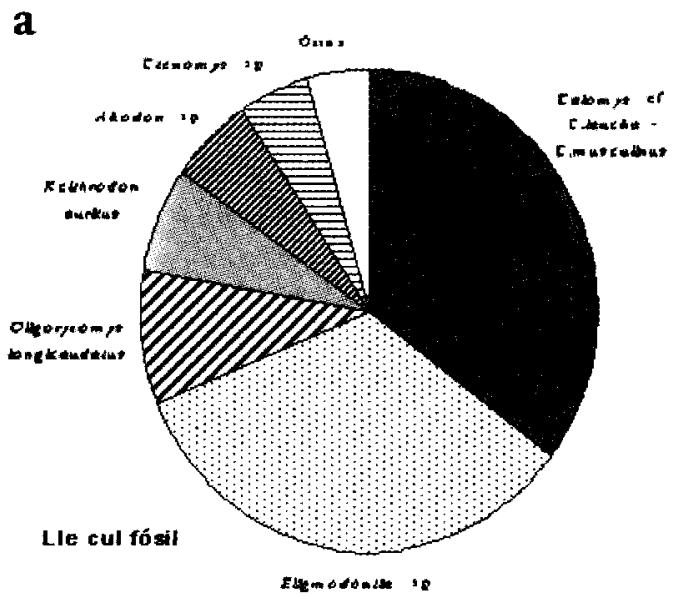

\section{Diversidad}

La muestra fósil de Lle cul presenta una composición taxonómica cualitativa similar a la actual de la misma localidad (Tabla 2). Sólo el marmosino Lestodelphys halli y el quiróptero Tadarida brasiliensis no se verifican entre los micromamíferos depredados hoy día por T. alba. Sin embargo, las diferencias cuantitativas entre am-

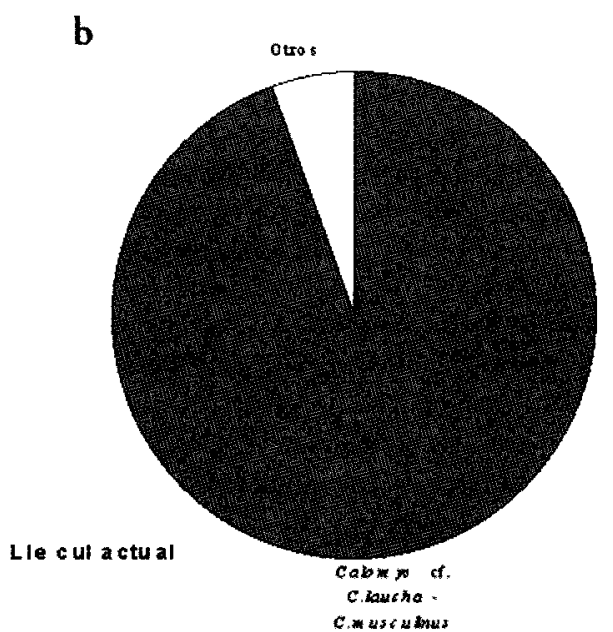

Fig. 5. Comparación taxonómica entre la muestra fósil (a) y actual (b) de Lle cul (Chubut, Argentina). Se indica MNI\% para cada taxa.

Taxonomic comparison between fossil (a) and recent (b) samples from Lle cul (Chubut, Argentina). MNI\% for each taxa is given. 
TABLA 3

Frecuencia esqueletaria absoluta en la muestra fósil de Lle cul (Chubut, Argentina) y en otras muestras utilizadas en la comparación (véase el texto)

Absolute skeletal frequency of the fossil sample from Lle cul (Chubut, Argentina) and of the other samples used in the comparison (see the text)

\begin{tabular}{lccccc}
\hline Elemento & Lle cul fósil & T. alba (disgregado) & A. flammeus & T. alba & B. bubo \\
\hline Hemimandíbula & 640 & 240 & 73 & 719 & 82 \\
Hemimaxilar & 463 & 146 & 41 & 485 & 36 \\
Escápula & 10 & 29 & 21 & 451 & 25 \\
Húmero & 371 & 186 & 70 & 549 & 64 \\
Radio & 50 & 90 & 48 & 508 & 30 \\
Cúbito & 103 & 142 & 50 & 554 & 59 \\
Coxal & 339 & 116 & 40 & 546 & 56 \\
Fémur & 612 & 225 & 57 & 573 & 56 \\
Tibia & 522 & 202 & 56 & 530 & 454 \\
NISP Total & 3.110 & 1.376 & 456 & 5.015 & 414 \\
MNI & 339 & 134 & 39 & & \\
\hline
\end{tabular}

bas muestras son importantes (Fig. 5) y no pueden ser explicadas por problemas de tamaño muestral o sesgos tafonómicos.

En el agregado fósil, 6 micromamíferos tienen porcentajes mayores al $5 \%$, dominando con frecuencias similares Calomys cf. C. laucha-C. musculinus y Eligmodontia sp. (Fig. 5a). En la muestra actual, casi el $95 \%$ está constituido por Calomys cf. C. laucha-C. musculinus (Fig. 5b). Estas diferencias quedan reflejadas en los valores del Indice de Diversidad de Shannon y Weaver (Tabla 4), que para la muestra fósil es de 0,690 mientras que para la actual, 0,121.

En los dos fenogramas resultantes entre la comparación de los micromamíferos de Lle cul fósil y análisis de egagrópilas actuales (Fig. 6), la mayor división recae entre las muestras del ecotono bosque-estepa (Trévelin y Río Corintos) y las de la estepa patagónica sensu lato. La topología del grupo de estepa para ambos fenogramas no refleja, en líneas generales, las divisiones fitogeográficas clásicamente reconocidas.

En el análisis por presencia-ausencia (Fig. 6a), las muestras fósil y actual de Lle cul forman un núcleo con alto índice de similitud, producto de una estructura taxonómica cualitativa prácticamente idéntica. A estas se asocia otra muestra del Monte (Laguna Blanca-Punta Este), pero con un valor de similitud claramente menor. La muestra de Meseta Lehman, correspondiente al Distrito

TABLA 4

Indice de diversidad (Shannon y Weaver), de equitatibilidad y número de especies (Nsp; excluyendo quirópteros) de la muestra fósil y actual de Lle cul y de los análisis de egagrópilas de Tyto alba (véase la Fig. 1) utilizados en la discusión

Species diversity (Shannon \& Weaver Index), evenness, and number of species (Nsp; bats excluding) of the fossil and recent sample from Lle cul (Chubut, Argentina) and of the other samples of owl pellets of Tyto alba (see Fig. 1) used in the discussion

\begin{tabular}{lccc}
\hline Muestra & Indice & Equitatividad & Nsp \\
\hline Estancia El Gauchito & 0,696 & 0,730 & 9 \\
Laguna Blanca-Punta Este & 0,584 & 0,646 & 8 \\
Lle cul actual & 0,121 & 0,127 & 9 \\
Lle cul fósil & 0,690 & 0,690 & 10 \\
Meseta Lehman & 0,087 & 0,145 & 4 \\
Río Corintos & 0,462 & 0,594 & 6 \\
Trévelin & 0,702 & 0,778 & 8 \\
Valle Hermoso & 0,778 & 1,000 & 6 \\
\hline
\end{tabular}




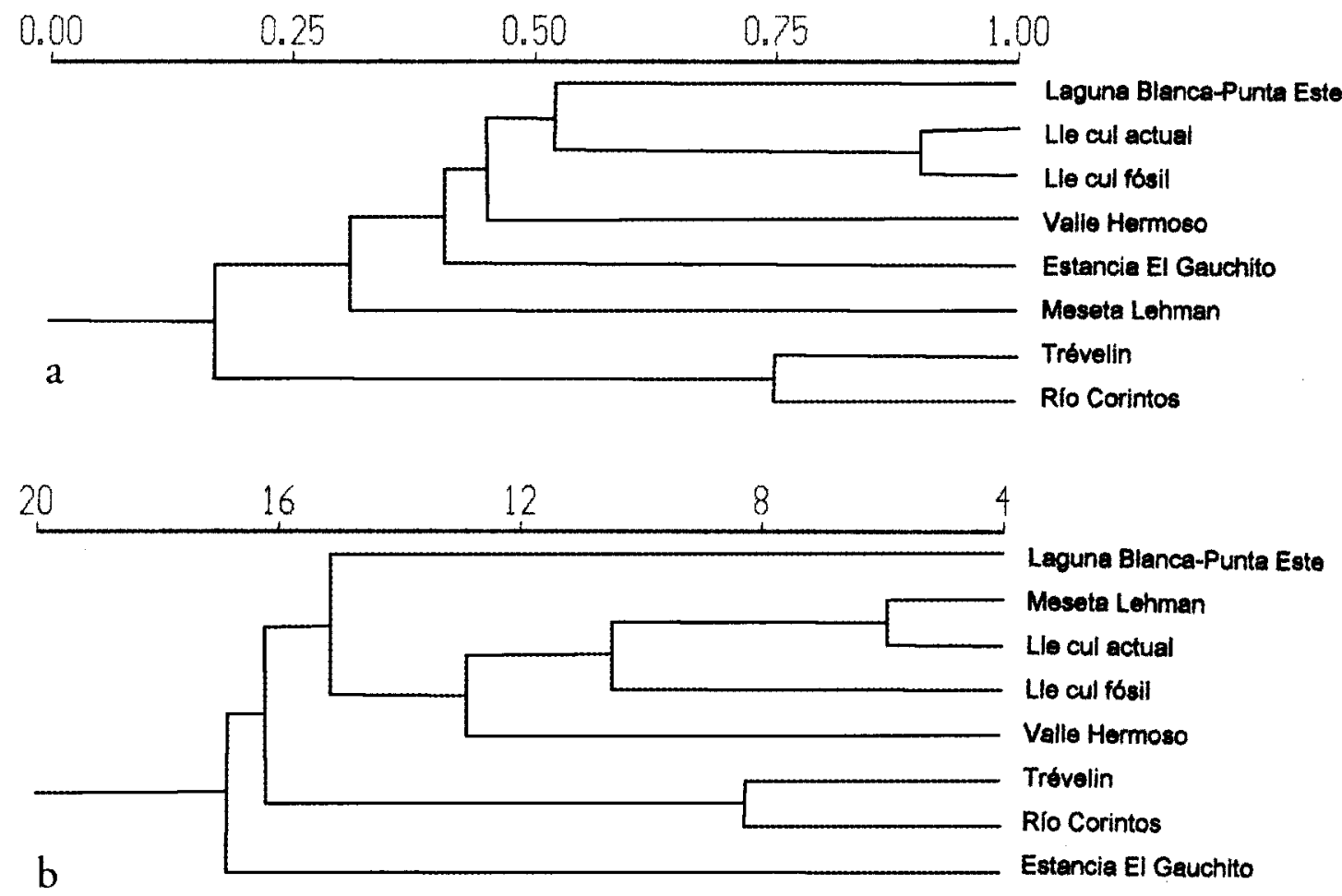

Fig. 6. Relaciones fenéticas entre la muestra fósil de Lle cul y los análisis de egagrópilas actuales para la provincia de Chubut (Argentina): a) Fenograma sobre la matriz de presencia-ausencia (coeficiente "Jaccard", CCC = 0,932), b) Fenograma sobre la matriz de frecuencias absolutas estandarizadas (coeficiente "distancia euclideana", $\mathrm{CCC}=0,930$ ).

Comparison of cluster diagrams of fossil sample of Lle cul and the recent owl pellets from Chubut Province (Argentina): a) Cluster based on presence-absence matrix ("Jaccard" coefficient, correlation coefficient $=0.932$ ); b) Cluster based on absolute standarized frequencies matrix ("Euclidean distances" coefficient, correlation coefficient $=0.930$ ).

Occidental, es la que más se aleja de las muestras de estepa. El resto de los agregados se agrupa independientemente de pertenecer al Distrito Central o a la región del Golfo San Jorge.

El análisis por frecuencias se ajusta de una manera más precisa con las divisiones fitogeográficas (Fig. 6b). La topología del fenograma muestra tres grupos discretos, que coinciden con las divisiones ecotono bosque-estepa (Trévelin + Río Corintos), distrito del Golfo San Jorge (Estancia El Gauchito) y Monte-Distrito Central (muestras restantes). En este último grupo se ha roto el núcleo entre Lle cul actual y Lle cul fósil.

\section{DISCUSION Y CONCLUSIONES}

\section{Micromamíferos}

La taxonomía de las especies del género Akodon que habitan en el norte de Patagonia es confusa. Para la región de estudio se han citado A. iniscatus Thomas, 1919 y A. molinae Contreras, 1968 (véa- se Daciuk 1974). El morfotipo de la muestra fósil -así como el registrado en la actualidad- no se corresponde con ninguna de las citadas, aunque sus caracteristicas lo acercan al "grupo varius" (sensu Myers 1989). La asignación a Oligoryzomys longicaudatus sigue un criterio de parsimonia, ya que las diferencias morfológicas entre esta especie y $O$. magellanicus (Bennett, 1836) no son adecuadamente conocidas (véase Gallardo \& Palma 1990). En cuanto a Calomys, la asignación específica es dudosa, ya que la diferenciación entre $C$. laucha (Fischer, 1814) y C. musculinus (Thomas, 1913) con restos craneanos fragmentarios no cuenta con criterios morfológicos sólidos (véase la discusión en Pardiñas \& Lezcano 1995). Aún así, para la región patagónica se ha citado únicamente C. musculinus (véase Massoia \& Fornes 1966). Para Eligmodontia se han descrito dos citotipos en la región patagónica, identificados con las formas nominales E. typus F. Cuvier, 1837 y E. morgani (Allen, 1901). Las diferencias morfológicas entre ambas especies no han sido evaluadas en detalle (véase Galliari et al. 1996). 
Un reciente análisis morfométrico (Sikes et al. 1997) indica que sólo es posible su separación mediante análisis discriminantes de varias medidas; según Hillyard et al. (1997) en la región de estudio únicamente se registra E. typus. Para las más de 60 especies descriptas del género Ctenomys las diferencias cráneo-dentarias no son bien conocidas. En el área de Gaiman se han citado $C$. mendocinus Philippi, 1869 y C. haigi Thomas, 1919. Finalmente, Birney et a1. (1996) reconocen la existencia de dos morfotipos del género Thylamys en Patagonia, que asimilan con las formas nominales $T$. pallidior (Thomas, 1902) y $T$. pusillus (Desmaret, 1804) distribuidas en las regiones del monte y estepa, respectivamente. Sin embargo, no hay un estudio a nivel cráneo-dentario que permita la diferenciación de estos taxa con restos fragmentarios.

\section{Deterioro ambiental}

Monjeau et al. (1997) a base de un análisis de presencia-ausencia de micromamíferos del centro-norte de Patagonia encuentran que los ensambles comunitarios son similares para diferentes asociaciones florísticas menores de la estepa patagónica. Esto implica que usar métodos de presencia-ausencia tendría poca efectividad en la reconstrucción paleoambiental. Nuestros resultados sugieren que las frecuencias estandarizadas reflejan más precisamente las divisiones fitogeográficas finas. Este comportamiento podría estar respondiendo a que las comunidades de micromamíferos de la estepa patagónica se insertan en una región con gradientes ambientales muy amplios, existiendo diferencias de frecuencias en función de variables ambientales de pequeña escala.

Existen muy pocos datos sobre la relación entre la cobertura y calidad vegetal con los micromamíferos en la región de estudio. Como una aproximación puede utilizarse los datos de Pearson (1995) obtenidos en tres décadas de trabajo en el noroeste de Patagonia. Eligmodontia morgani es un típico sigmodontino de la estepa arbustiva y semidesierto patagónico, cuya densidad disminuye notablemente con el sobrepastoreo (Pearson et al. 1987). Reithrodon auritus habita pastizales densos -pero de bajo porte- en áreas cercanas a vegas y praderas herbáceas, incluyendo campos con pasturas introducidas. Oligoryzomys longicaudatus es frecuente en la estepa arbustiva, con mediano porcentaje de suelo desnudo. Por su parte Calomys es el roedor más abundante en agroecosistemas de la región pampeana (Bilenca \& Kravetz 1995). Graomys griseoflavus se asocia al monte arbustivo y espinal en la región central de Argentina, situación similar a la de Microcavia australis, cuya abundancia y distribución parecen estar relacionadas con microhábitats específicos de arbustos (véase Tognelli et al. 1995).

Los resultados obtenidos brindan soporte a la hipótesis de trabajo planteada inicialmente. Las diferencias cuantitativas observadas entre las muestras actual y fósil de Lle cul parecen responder a un deterioro del hábitat ocurrido por procesos fundamentalmente antrópicos. Los estudios paleoambientales llevados a cabo en el nordeste de Patagonia indican el establecimiento de condiciones climáticas similares a las actuales hacia los 3,5 a 2,5 ka (véase Schäbitz 1991, Garleff et al. 1994). Hacia los $1,8 \mathrm{ka}$ la comunidad de micromamíferos del valle inferior del río Chubut ya estaba integrada por los mismos taxa que se registran en la actualidad, indicando una vegetación local del tipo estepa arbustiva y herbácea con elementos de monte. Factores antrópicos predominantes durante los últimos 100 años, ganadería ovina extensiva y sobrepastoreo, establecimiento de cultivos, etc. (véase Ares et al. 1996, Del Valle et al. 1995, Soriano 1956b, Soriano \& Paruelo 1990), habrían provocado una homogeneidad de microambientes transformando parcialmente la región en un agroecosistema. De hecho, el valle inferior del río Chubut ha tenido un desarrollo importante con el establecimiento de chacras, el cultivo de alfalfa y frutales, especialmente a partir de la implementación de regadío. El crecimiento de la agricultura se reflejaría en la dieta de $T$. alba en un marcado incremento de Calomys cf. C. laucha-C. musculinus, un sigmodontino oportunista dominante en ambientes antropogénicos (véase Mills et al. 1991b, Bellocq 1990). Otras especies de roedores quedarían prácticamente restringidas a escasos "parches" (bordes de campos cultivados, bordes de canales, márgenes de cuerpos de agua) donde la vegetación conservaría sus características naturales.

Las conclusiones de este trabajo pueden extrapolarse a otras localidades de Patagonia, seriamente afectadas por la expansión de los agroecosistemas y el sobrepastoreo. En el fenograma de la Fig. 6b, la unión entre Lle cul actual y Meseta Lehman es sumamente interesante si se tiene en cuenta que en esta última el grado de modificación antrópica de los ambientes naturales es muy elevado (Indice de Diversidad = 0,087 ), debido a la acción combinada del sobrepastoreo y un clima muy árido (véase Ares et al. 1995). En esta localidad, la frecuencia de Calomys cf. C. laucha-C. musculinus supera el $95 \%$ (Tabla 1). Unas pocas décadas atrás los registros más australes para el género Calomys alcanzaban sólo el norte del río Colorado (véase 
Hershkovitz 1962). Trampeos y análisis de egagrópilas han demostrado una extensa distribución de este roedor en la Patagonia, llegando hasta el pie de cordillera en la provincia de Santa Cruz (véase Massoia \& Pardiñas 1993).

Finalmente, es importante destacar que tanto $C$. laucha como $C$. musculinus son vectores de zoonosis -virus Junín o Fiebre Hemorrágica Argentina, virus Hantaan, etc.- en la región pampeana (véase Mills et al. 1991a, 1994). Comprender las causales históricas de su distribución y dominancia puede convertirse en una herramienta para el control y predicción de tales enfermedades ${ }^{1}$. Los resultados obtenidos en este trabajo sugieren su utilidad como un indicador de impacto antrópico en la estepa patagónica.

\section{AGRADECIMIENTOS}

G. Pagnoni recolectó la muestra fósil y F. Goin ratificó las determinaciones sobre marmosinos. Dos revisores anónimos contribuyeron con sus críticas a mejorar esta contribución. Este trabajo fue parcialmente solventado con fondos del Consejo Nacional de Investigaciones Científicas y Técnicas y de la Fundación FOSDIC (becas a U. Pardiñas) y de la Universidad Nacional La Plata (Proyecto \# N180). A todas las personas e instituciones mencionadas, el reconocimiento de los autores, únicos responsables de lo expresado.

\section{LITERATURA CITADA}

ANDREWS P (1990) Owls, Caves and Fossils. Predation, Preservation, and Accumulation of small mammal bones in caves, with an analysis of the Pleistocene Cave faunas from Westbury-sub-Mendip, Somerset, UK. University of Chicago Press, Chicago. 231 pp.

ARES J, AM BEESKOW, M BERTILLER, M ROSTAGNO, M IRISARRI, J ANCHORENA, G DEFOSSE \& C MERINO (1995) Structural and Dynamic characteristics of overgrazed lands of northern Patagonia, Argentina. En: Breymeyer A (ed) Managed Grasslands: 149-175. Elsevier Science Publishers B.V., Amsterdam.

BEESKOW AM, HF DEL VALLE \& CM ROSTAGNO (1987) Los sistemas fisiográficos de la región árida y semiárida de la provincia de Chubut. CECYT, San Carlos de Bariloche. 144 pp.

BELLOCQ MI \& FO KRAVETZ (1983) Identificación de especies, sexo y edad relativa a partir de restos óseos de roedores de la provincia de Buenos Aires, Argentina. Historia Natural (Corrientes) 3: 101-112.
BELLOCQ MI (1990) Composición y variación temporal de la dieta de Tyto alba en ecosistemas agrarios pampeanos, Argentina. Vida Silvestre Neotropical 2: 32-35.

BILENCA DN \& FO KRAVETZ (1995) Patrones de abundancia relativa en ensambles de pequeños roedores de la región pampeana. Ecología Austral 5: 21-30.

BIRNEY EC, RS SIKES, JA MONJEAU, N GUTHMANN \& CJ PHILLIPS (1996) Comments on Patagonian marsupials of Argentina. En: Contributions in Mammalogy, a Memorial Volume honoring Dr. J. Knox Jones Jr.: 149-154, Museum of Texas Tech University.

CLAPPERTON CM (1993) Quaternary Geology and Geomorphology of South America. Elsevier Science Publishers B.V., Amsterdam 779 pp.

COLVIN BA \& EB Mc LEAN (1986) Food habits and prey specificity of the Common Barn Owl in Ohio. Ohio Journal Science 86: 76-80.

CRISCI JV \& MF LOPEZ ARMENGOL (1983) Introducción a la teoría y práctica de la taxonomía numérica. Monografías de la OEA, Serie Biología 26: 1-129.

DACIUK J (1974) Notas faunísticas y bioecológicas de Península Valdés y Patagonia. XII. Mamíferos colectados y observados en la Península Valdés y zona litoral de los golfos San José y Nuevo (provincia de Chubut, República Argentina). Physis, sección C 33: 23-39.

DE SANTIS LJM \& GO PAGNONI (1989) Alimentación de Tyto alba (Aves: Tytonidae) en localidades costeras de la Provincia del Chubut (Argentina). Neotrópica 35: 43-49.

DE SANTIS LJM, CM GARCIA ESPONDA \& GJ MOREIRA (1996) Vertebrados depredados por Tyto alba (Aves: Tytonidae) en el Sudoeste de la provincia del Chubut (Argentina). Neotrópica 42: 123.

DE SANTIS LJM, IM PEÑA-COZZARIN \& MF GROSMAN (1993) Vertebrados depredados por Tyto alba (Aves: Tytonidae) en las proximidades del río Corintos (Provincia del Chubut, Argentina). Neotrópica 39: 53-54.

DE SANTIS LJM, MF TEJEDOR \& MF GROSMAN (1991) Vertebrados contenidos en egagrópilas de Tyto alba (Aves: Tytonidae) para el área precordillerana del Chubut (República Argentina). Neotrópica 37: 24.

DE SANTIS LJM, NG BASSO, JI NORIEGA \& MF GROSMAN (1994) Explotación del recurso trófico por la lechuza de los campanarios (Tyto alba) en el oeste de Chubut, Argentina. Studies in Neotropical Fauna \& Environment 29: 43-47.

DEL VALLE HF, JC LABRAGA \& J GOERGEN (1995) Biozonas de la región patagónica. En: Proyecto INTAGTZ (eds) Evaluación del estado actuales de la desertificación en áreas representativas de la Patagonia: informe final de la etapa I: 33-55. Puerto Madryn.

FAVERIN C (1987) Variación de la dieta de la lechuza del campanario (Tyto alba) en Lobos, provincia de Buenos Aires. Seminario de Licenciatura, Universidad Nacional de Mar del Plata. 98 pp.

\footnotetext{
' PARDIÑAS UFJ (1998) Roedores y cambios climático-ambientales durante los últimos 10.000 años en la región pampeana (Argentina). En: $2^{\text {do }}$ Congreso Argentino de Zoonosis y $1^{\text {er }}$ Congreso Argentino y Latinoamericano de Enfermedades Emergentes (eds) Temas de zoonosis y enfermedades emergentes: 205-208, Buenos Aires.
} 
FERNANDEZ-JALVO Y \& P ANDREWS (1992) Small mammal taphonomy of Gran Dolina, Atapuerca (Burgos), Spain. Journal of Archaeological Science 19: 407-428.

GALLARDO M \& E PALMA (1990) Systematics of Oryzomys longicaudatus (Rodentia: Muridae) in Chile. Journal of Mammalogy 71: 333-342.

GALLIARI C, UFJ PARDIÑAS \& FJ GOIN (1996) Lista comentada de los mamíferos argentinos. Mastozoología Neotropical 3: 39-61.

GALLOWAY R, V MARKGRAF \& JP BRADBURY (1988) Dating shorelines of lakes in Patagonia, Argentina. Journal of South American Earth Sciences 1: 195-198.

GARCIA-ESPONDA CM, LJM DE SANTIS, JI NORIEGA, GO PAGNONI, GJ MOREIRA \& M BERTELLOTI (1998) The diet of Tyto alba (Strigiformes, Tytonidae) at the lower valley of the Chubut river (Chubut Province, Argentina). Neotrópica 44: 57-63.

GARLEFF K, T REICHERT, M SAGE, F SCHÄBITZ \& B STEIN (1994) Períodos morfodinámicos y el paleoclima en el norte de la Patagonia durante los últimos 13.000 años. 2nd Ann. Meeting Project 341 IGCP/IUGS UNESCO. Revista del Museo de Historia Natural de San Rafael 12: 179.

GAUCH HG (1982) Multivariate Analysis in Community Ecology. Cambridge Univ. Press, New York. 432 pp.

GRAYSON DK (1973) On the methodology of faunal analysis. American Antiquity 39: 432-439.

HERSHKOVITZ P (1962) Evolution of Neotropical Cricetine Rodents (Muridae) with special reference to the phyllotine group. Fieldiana, Zoology 46: 1-524.

HEUSSER CJ, J RABASSA, A BRANDANI \& R STUCKENRATH (1988) Late-Holocene vegetation of the Andean Araucaria region, Province of Neuquén, Argentina. Mountain Research and Development 1: 53-63.

HILLYARD J, C PHILLIPS, E BIRNEY, J MONJEAU \& R SIKES (1997) Mitochondrial DNA analysis and zoogeography of two species of silky desert mice, Eligmodontia, in Patagonia. Zeitschrift für Säugetierkunde 62: 282-292.

HOFFMAN R (1988) The contribution of raptorial birds to patterning in small mammal assemblages. Paleobiology 14: 81-90.

IRIONDO M (1995) Patagonia extraandina y Tierra del Fuego. En: Argollo J \& P Mourguiart (eds) Los climas cuaternarios en América del Sur: 339-344. ORSTOM, La Paz.

JAKSIC F \& J YAÑEZ (1979) The diet of the Barn Owls in Central Chile and its relation to the availability of prey. The Auk 97: 895-896.

LYMAN RL (1994) Relative abundances of skeletal specimens and taphonomic analysis of vertebrate remains. Palaios 9: 288-298.

MARKGRAF V (1993) Climatic history of central and South America since 18,000 yr B.P.: comparison of pollen records and model simulations. En: Wright HE, JE Kutzbach, T Webb, WF Ruddiman, FA StreetPerrott \& PJ Bartlein (eds) Global climates since the Last Glacial Maximum: 357-387. University of Minnesota Press, Minnesota.
MARKGRAF V, JW WHITE, R FIGGE \& R KENNY (1995) Multivariate climate reconstruction for the last 14,000 years in southernmost South America. Proceedings of the 11 th Annual Pacific Climate, Workshop, Interagency Ecological Program, California Water Resources. Technical Report 40: 21-28.

MASSOIA E \& A FORNES (1966) Nuevos datos sobre la distribución geográfica y ecología del género Calomys (Waterhouse) (Rodentia-Cricetidae). Idia 227: 5557.

MASSOIA E \& UFJ PARDIÑAS (1988) Nota sobre la fauna de pequeños roedores de Valle Hermoso, Departamento Escalante, Provincia de Chubut. Asociación para la Protección de la Naturaleza, Boletín Científico 11: 13-15.

MASSOIA E \& UFJ PARDIÑAS (1993) La depredación de mamíferos por Bubo virginianus y Tyto alba en Cerro Casa de Piedra, Lago Burmeister, Parque Nacional Perito Moreno, provincia de Santa Cruz. Asociación para la Protección de la Naturaleza, Boletín Científico 26: 6-12

MILLS JN, GE CALDERON, BA ELLIS, KT McKEE, TG KSIAZEK, JG BARRERA ORO, C J PETERS, JE CHILDS \& JI MAIZTEGUI (1991a) Nuevas observaciones de la infección de roedores por el virus Junín dentro y fuera de la zona endémica de la fiebre hemorrágica argentina. Medicina 51: 519-523.

MILLS JN, BA ELLIS, KT McKEE, JI MAIZTEGUI \& JE CHILDS (1991b) Habitat associations and relative densities of rodent populations in cultivated areas of Central Argentina. Journal of Mammalogy 72: 470479.

MILLS JN, JECHILDS, DA ENRIA, MD BOWEN, CJ PETERS, TG KSIAZEK \& PB JAHRLING (1994). Oliveros virus: seroprevalence of a new arenavirus in rodents and humans in central Argentina. American Journal of Tropical Medicene and Hygiene 51: 96-97.

MIOTTI L (1993) La ocupación humana de la Patagonia austral durante el Holoceno. En: Iriondo $M$ (ed) El Holoceno en la Argentina, Volumen 2: 94-130. CADINQUA, Corrientes.

MONJEAU JA, N BONINO \& S SABA (1994) Annotated checklist of the living land mammals in Patagonia, Argentina. Mastozoología Neotropical 1: 143-156.

MONJEAU J, R SIKES, E BIRNEY, N GUTHMANN \& C PHILLIPS (1997) Small mammal community composition within the major landscape divisions of Patagonia, Southern Argentina. Mastozoología Neotropical 4: 113-127.

MYERS P (1989) A preliminary revision of the varius group of Akodon (A. dayi, dolores, molinae, neocenus, simulator, toba y varius). En: Redford $\mathrm{KH} \& \mathrm{JF}$ Eisenberg (eds) Advances in Neotropical Mammalogy: 5-54. Sandhill Crane Press, Gainesville.

PARDIÑAS UFJ (1998) Roedores holocénicos del sitio Cerro Casa de Piedra 5 (Santa Cruz, Argentina): tafonomía y paleoambientes. Palimpsesto 5: 66-90.

PARDIÑAS UFJ \& MJ LEZCANO (1995) Cricétidos (Mammalia, Rodentia) del Pleistoceno tardío del nordeste de la provincia de Buenos Aires (Argentina). Aspectos sistemáticos y paleoambientales. Ameghiniana 32: 249-265. 
PEARSON OP (1995) Annotated keys for identifying small mammals living in or near Nahuel Huapi National Park or Lanín National Park, southern Argentina. Mastozoología Neotropical 2: 99-148.

PEARSON O \& AK PEARSON (1993) La fauna de mamíferos pequeños cerca de Cueva Traful I, Argentina: pasado y presente. Praehistoria 1: 73-89.

PEARSON O, S MARTIN \& J BELLATI (1987) Demography and reproduction of the silky desert mouse (Eligmodontia) in Argentina. Fieldiana, Zoology, 39: 433-446.

PRIETO A \& S STUTZ (1996) Vegetación del Holoceno en el norte de la estepa patagónica: palinología de la cueva Epullán Grande (Neuquén). Praehistoria 2: 267 278.

ROLFH FJ (1993) NTSYS-pc- Numerical Taxonomy and multivariate analysis system, version 1.8. Exeter Software: Setauket, New York.

SAAVEDRA B \& JA SIMONETTI (1998) Small mammal taphonomy: intraspecific bone assemblage comparison between South and North American barn owl, Tyto alba, populations. Journal of Archaeological Science 25: $165-170$.

SAINT GIRONS MC (1973) L'age des micromammiferes dans le régime de deux rapaces nocturnes, Tyto alba et Asio otus. Mammalia 37: 439-456.

SCH ̈̈BITZ F (1991) Paleoecological studies of the "bajos sin salida" of northern Patagonia (Laguna Indio Muerto), Argentina (Preliminary results). Bamberger Geographische Schriften 11: 295-308.

SCHÄBITZ F (1994) Holocene climatic variations in northern Patagonia, Argentina. Palaeogeography, Palaeoclimatology, Palaeoecology 109: 287-294.

SIKES R, A MONJEAU, E BIRNEY, C PHILLIPS \& J HILLYARD (1997) Morphological versus chromosomal and molecular divergence in two species of Eligmodontia. Zeitschrift für Säugetierkunde 62: 265280 .
SIMONETTI JA \& JR RAU (1989) Roedores del Holoceno Temprano de la Cueva del Milodón, Magallanes, Chile. Noticiario Mensual, Museo Nacional de Historia Natural 315: 3-5.

SORIANO A (1956a) Los distritos florísticos de la Provincia Patagónica. Revista de Investigaciones Agrícolas 10: 323-348.

SORIANO A (1956b) Aspectos ecológicos y pasturiles de la vegetación patagónica relacionados con su estado y capacidad de recuperación. Revista de Investigaciones Agrícolas 10: 349-372.

SORIANO A \& JM PARUELO (1990) El pastoreo ovino. Principios ecológicos para el manejo de los campos. Ciencia Hoy 2: 44-53.

SORIANO A, CP MOVIA \& RJC LEÓN (1983) Vegetation. En: West EN (ed) Temperate Desert and Semi-Deserts: 440-454. Elsevier, Amsterdam.

STAHL PW (1996) The recovery and interpretation of microvertebrate bone assemblages from archaeological contexts. Journal of Archaeological Method and Theory 3: $31-75$.

STINE S \& M STINE (1990) A record from lake Cardiel of climate change in southern South America. Nature 345: 705-708.

STRAUSS RE (1982) Statistical significance of species clusters in association analysis. Ecology 63: 634639.

TOGNELLI MF, CM CAMPOS, RA OJEDA \& VG ROIG (1995) Is Microcavia australis (Rodentia: Caviidae) associated with a particular plant structure in the Monte desert of Argentina? Mammalia 59: 327-333.

VILLALBA R (1995) Geographical variations in treegrowth responses to climate in the southern Andes. En: Argollo J \& P Mourguiart (eds) Los climas cuaternarios en América del Sur: 307-318. ORSTOM, La Paz.

ZAMORANO E, L PALOMO, A ANTUÑEZ \& J VARGAS (1986) Criterios de predación selectiva de $B u b o$ bubo y Tyto alba sobre Rattus. Ardeola 33: 3-9.

APENDICE

Matriz de similitud (Coeficiente: Jaccard)

$\begin{array}{lll}\text { Laguna Blanca-Punta Este } & 1,000 & \\ \text { Trevelin } & 0,142 & 1,000 \\ \text { Río Corintos } & 0,166 & 0,750 \\ \text { Meseta Lehman } & 0,200 & 0,090 \\ \text { Valle Hermoso } & 0,400 & 0,166 \\ \text { Lle cul actual } & 0,545 & 0,214 \\ \text { Lle cul fósil } & 0,500 & 0,200 \\ \text { Estancia El Gauchito } & 0,416 & 0,133\end{array}$

1,000

$0,111 \quad 1,000$

$0,200 \quad 0,428$

$0,250 \quad 0,444$

$0,230 \quad 0,400$

$0,153 \quad 0,083$

1,000

$0,500 \quad 1,000$

$0.454 \quad 0,900$

0,363

$0,384 \quad 0,461$

1,000

Matriz de distancia (Coeficiente: distancia euclideana)

Laguna Blanca-Punta Este
Trevelin
Río Corintos
Meseta Lehman
Valle Hermoso
Lle cul actual
Lle cul fósil
Estancia El Gauchito

0,000

$17,146 \quad 0,000$

$15,556 \quad 8,246$

$17,000 \quad 17,464$

$14,456 \quad 18,357$

$14,352 \quad 16,186$

$14,662 \quad 15,066$

$16,062 \quad 17,776$
0,000

16,217

17,464

14,282

14,317

16,970
0,000

13,856

5,916

11,313

17,635
0,000

12,767

11,916

14,730

0,000

9,643

$16,673 \quad 17,748$ 